\title{
Criterion for existence of the negative dielectric reality in crystals
}

\author{
Stanislav Felinskyi, Georgii Felinskyi \\ Kyiv National Taras Shevchenko University, Faculty of Radiophysics, Electronics and Computer Systems, Kyiv, Ukraine
}

Email address:

felinskyi.sg@gmail.com (S. G. Felinskyi), felinskyi@yahoo.com (G. S. Felinskyi)

To cite this article:

Stanislav Felinskyi, Georgii Felinskyi. Criterion for Existence of the Negative Dielectric Reality in Crystals. American Journal of Modern Physics. Vol. 3, No. 5, 2014, pp. 195-201. doi: 10.11648/j.ajmp.20140305.11

\begin{abstract}
The real physical conditions of appearance the negative dielectric permittivity (DP) within frequency T-L splitting of polar vibrations in crystals are discussed in this paper. We have derived the simple quantitative criterion for the existence of negative DP band using single oscillation model. Our criterion is presented in three equivalent forms as inequalities between the fundamental crystal constants and spectroscopic parameters of polar vibration. Applicability of our theoretical results for multimode case is demonstrated using 7 known polar vibrations in the model crystal of boron nitride.
\end{abstract}

Keywords: Negative Dielectric Permittivity, Metamaterials, Waves Reflection, Residual Ray Band

\section{Introduction}

The fundamental features of electromagnetic waves propagation in condensed media today are the subject of heightened researcher's attention. Most efforts are concentrated on the anomalous dispersion areas in materials. There are the negative values of dielectric and/or magnetic permittivity may be accumulated only in the anomalous dispersion regions. Deepen interest to the negative values of the material parameters is explained by the part of such extraordinary electrodynamics of "left-sided" media [1-3] now named as metamaterials [4]. In addition the phenomenal perspectives are promised by the metamaterials synthesis: from fundamentals of transformation optics [5-7] to design various functional devices, including invisible cloaks [8,9], perfect lens [10], etc.

It should be noted the occurrence of negative dielectric values due to anomalous dispersion wasn't the new phenomenon and it is recorded by many experts in the field of vibrational spectroscopy of crystals.

However, the analysis of the physical conditions leading to the presence or absence of negative dielectric permittivity (DP) doesn't made jet. In particular the wave attenuation effects inside T-L frequency splitting of polar oscillation previously have almost always been neglected. The wave dumping in the photon-phonon interaction theory [2] as well as in the metamaterials $[5,7]$ optics is considered as a harmful but the secondary factor. It is assumed that the polariton damping does not cause any physical consequences and attenuation influence may be eliminated by a simple gain of the light wave. Such approach is quite reasonable in the transparency of the crystal but it is inapplicable in the anomalous dispersion region where the imaginary part of the DP $\varepsilon^{\prime \prime}$ not only quit being negligible and it may greatly exceed the real permittivity part $\varepsilon^{\prime}$. Actual negative DP limits as function of phonon dumping are obtained in our work.

\section{Problem Statement and Theoretical Base}

The start point of the quantitative theory of photon-phonon interaction may be taken using the well-known Lyddane-Sachs-Teller (LST) [11] ratio:

$$
\frac{\omega_{L}^{2}}{\omega_{T}^{2}}=\frac{\varepsilon_{0}}{\varepsilon^{\infty}},
$$

where $\omega_{L}$ and $\omega_{T}$ are accordingly the longitudinal and transverse vibration frequencies in the single fundamental mode case; $\varepsilon_{0}$ and $\varepsilon^{\infty}$ are dielectric constants in the limit points on the dispersion dependence of DP $\varepsilon(\omega)$, respectively, at $\omega \rightarrow 0$ and $\omega \rightarrow \infty$.

Formation of modern conceptions of alliance between crystal spectroscopic and material parameters is based on 
approximation of the phonon damping absence $\Gamma_{i}=0$, where index $i$ denotes the count of separate mode.

Using no dumping restriction the main values of DP tensor components with polarization along the $k^{\text {th }}$ axis can be expressed in general form through the frequencies of longitudinal $\omega_{i k}^{L}$ and transverse $\omega_{i k}^{T}$ vibrations as Kurosawa [12] equation:

$$
\varepsilon_{k}(\omega)=\varepsilon_{k}^{\infty} \prod_{i=1}^{N_{k}} \frac{\left(\omega_{i k}^{L}\right)^{2}-\omega^{2}}{\left(\omega_{i k}^{T}\right)^{2}-\omega^{2}}
$$

which describes the DP frequency dispersion in the damping absence.

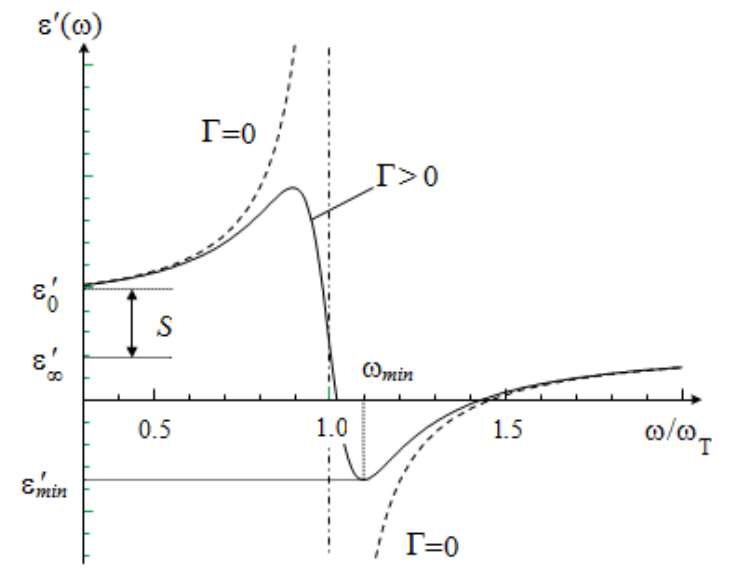

Fig 1. The real part of the dielectric function of frequency around the polar oscillation resonance at the absence of damping (dotted line) and for the normalized attenuation constant $\Gamma / \omega T=0.2$ (solid line).

It should be noted the Eq. (2) guarantees the existence of a negative DP for arbitrary polar lattice vibrations in the frequency range of $\omega_{i k}^{T}<\omega<\omega_{i k}^{L}$, i.e., in T-L splitting also known as residual ray band [13].

The last conclusion remains valid in practice only for oscillations with large oscillator strength and, accordingly, with a large T-L-splitting of the phonon frequencies. Otherwise, if phonons damping is increased (it is observed with increasing of temperature) then the negative DP region is considerably narrowed and it may be fully disappeared.

Generally interaction of light with $N_{k}$ polar lattice vibrations of crystal is described within the semi-classical approach [14]. As result the dispersion dependence of DP tensor $\varepsilon_{k}(\omega)$ is determined by the common expression:

$$
\varepsilon_{k}(\omega)=\varepsilon_{k}^{\prime}(\omega)+i \varepsilon_{k}^{\prime \prime}(\omega)=\varepsilon_{k}^{\infty}+\sum_{j=1}^{N_{k}} \frac{S_{j k} \omega_{j k}^{2}}{\omega_{j k}^{2}-\omega^{2}-i \omega \Gamma_{j}},
$$

where $\omega_{j k}$ and $S_{j k}$ are respectively transverse phonon frequency and the oscillator strength of $j$-th lattice vibration with polarization along the $k$-th axis and the damping constant $\Gamma_{j} ; \varepsilon_{k}^{\infty}$ is the principal value of the dielectric tensor at optical frequencies.

One can to determine the oscillator strength directly by comparing Eq. (3) and (2) in $\Gamma_{\mathrm{i}}=0$ approximations as:

$$
S_{i}=\varepsilon^{\infty}\left[\left(\frac{\omega_{i}^{L}}{\omega_{i}^{T}}\right)^{2}-1\right] \prod_{j \neq i}^{N} \frac{\left(\omega_{j}^{L}\right)^{2}-\left(\omega_{i}^{T}\right)^{2}}{\left(\omega_{j}^{T}\right)^{2}-\left(\omega_{i}^{T}\right)^{2}},
$$

where the index $k$ to denote polarization is omitted to simplify the notation.

\subsection{Single Mode Model and Complex Refractive Index}

The DP real parts for a crystal with single polar oscillation (parameters $\varepsilon^{\infty}=2.3$ and $S=2.6$ are nearly closed to the model crystal $\mathrm{KBr}$ ) as functions of frequency are shown on Fig. 1. The case of normalized attenuation of $\Gamma / \omega_{T}==0.2$ is plotted by solid line. The dotted line shows the graph of the dielectric function (3) in the damping absence $(\Gamma=0)$. It is a damping of optical phonon eliminates the infinity interrupt of the dielectric function as shown in Fig. 1. In fact the anomalous dispersion of waves in a crystal should be observed only in case of $\Gamma>0$ where $d \varepsilon^{\prime}(\omega) / d \omega<0$. Moreover the negative DP region with $\varepsilon_{\text {min }}^{\prime}<0$ may exist only in case where $\Gamma$ does not exceed a certain critical value as we will obtain below.

The expression (3) for $\varepsilon(\omega)$ takes a most simple form within the limits of a single oscillator model. So the complex refractive index $\tilde{n}$ combined real part $n$ and extinction coefficient $\kappa$ as imaginary part in form $\tilde{n}=n-i \kappa$ (see for example [13]) and it may be written as:

$$
\tilde{n}^{2}(\omega)=\varepsilon(\omega)=\varepsilon^{\infty}+\frac{S \omega_{T}^{2}}{\omega_{T}^{2}-\omega^{2}-i \omega \Gamma} .
$$

The single mode equation (5) is the basis for the description of the electromagnetic waves reflection from the crystal in the phonon resonance area and it allows us to fully restore the dielectric function form using experimental data. Such numerical technique is widespread and it is well known as dispersion analysis (DA) of reflection spectra.

\subsection{Dispersion Analysis of Reflection Spectra}

Measured values of the reflection coefficient $R$ for normal incidence are expressed through a refractive index $n$ and extinction coefficient $\kappa$ as:

$$
R=\frac{(n-1)^{2}+\kappa^{2}}{(n+1)^{2}+\kappa^{2}} .
$$

Equation (6) proper describes the reflection spectrum $R(\omega)$ including frequencies around the polar oscillation resonance. Indeed both parts of $\tilde{n}$ are the functions of $\omega$ namely $n=$ $n(\omega)$ and $\kappa=\kappa(\omega)$ if one take into account the equation (5). So dispersion analysis technique is the fitting of experimental reflection spectrum by function $R(\omega)(6)$ using numerical methods and best fitting results are the set of parameters $S, \omega_{T}$, and $\Gamma$.

The universal DP form (3) should be used in eq. (5) for general DA procedure. Therefore obtained parameters $S_{j k}$, $\omega_{j k}$ and $\Gamma_{j}$ from spectroscopic measurements actually determines the form both function $\varepsilon^{\prime}(\omega)$ and $\varepsilon^{\prime \prime}(\omega)$ of the 
crystal including all anomalous dispersion regions. We have used the data [15] of general DA procedure for testing our results on the 7 measured vibrations in three modifications of boron nitride (cubic, orthorhombic and hexagonal). Single mode model allow us to obtain criterion for the existence of negative DP in the simple analytic form and next analysis of the physical conditions of proper regions formation with $\varepsilon^{\prime}(\omega)<0$ is the subject of this investigation.

\section{Criterion of the Negative DP Existence}

If the crystal has one vibration then common eq. (3) can be rewritten in the simplest form as:

$$
\varepsilon(\omega)=\varepsilon^{\infty}+\frac{S \omega_{T}^{2}}{\omega_{T}^{2}-\omega^{2}-i \omega \Gamma},
$$

where $S$ is the oscillator strength, $\varepsilon^{\infty}$ is the dielectric constant at high frequencies, $\omega_{T}$ is the fundamental own frequency of transverse vibrations, $\Gamma$ is the damping constant. Here the oscillator strength is defined as $S=\varepsilon_{0}-\varepsilon^{\infty}$ (see Fig.1), and $\varepsilon_{0}=\left.\varepsilon(\omega)\right|_{\omega \rightarrow 0}$ is the value of the dielectric function in the low-frequency region where $\omega \rightarrow 0$.

\subsection{Minimum DP Analysis}

Let the $\varepsilon(\omega)=\varepsilon^{\prime}(\omega)+i \varepsilon^{\prime \prime}(\omega)$ then the dielectric function (7) may be written in the following form:

$$
\left\{\begin{array}{l}
\varepsilon^{\prime}(\omega)=\varepsilon^{\infty}+\frac{S \omega_{T}^{2}\left(\omega_{T}^{2}-\omega^{2}\right)}{\left(\omega_{T}^{2}-\omega^{2}\right)^{2}+\omega^{2} \Gamma^{2}}, \\
\varepsilon^{\prime \prime}(\omega)=\frac{S \omega_{T}^{2} \omega \Gamma}{\left(\omega_{T}^{2}-\omega^{2}\right)^{2}+\omega^{2} \Gamma^{2}} .
\end{array}\right.
$$

Negative values, according to (8), takes only the real part of the DP $\varepsilon^{\prime}(\omega)$, but only in a limited range of values $\Gamma$ (Fig. $2 a)$. The imaginary part $\varepsilon^{\prime \prime}(\omega)$ is responsible for the absorption and it is always positive. Function $\varepsilon^{\prime}(\omega)$ reaches its extremes at frequencies where the condition:

$$
\frac{d \varepsilon^{\prime}}{d \omega}=0
$$

which by direct substitution of first equation (8) into (9) leads to the equation:

$$
\left(\omega_{T}^{2}-\omega^{2}\right)^{2}=\omega_{T}^{2} \Gamma^{2} .
$$

Minimum frequency position $\varepsilon^{\prime}(\omega)$ is determined by one of the equation (10) roots namely this root should be belonged to the frequency interval $\omega>\omega_{T}$. Therefore the minimum $\left(\omega_{\min }\right)$ point on frequency scale is located at:

$$
\omega_{\min }=\sqrt{\omega_{T}^{2}+\omega_{T} \Gamma},
$$

Substituting $\omega_{\min }$ into (8), we obtain the minimum value of the dielectric function $\varepsilon^{\prime}(\omega)$. The minimum frequency position and the minimum value $\varepsilon^{\prime}(\omega)$ according to (11) are noticeably depended on the damping parameter $\Gamma$ (see also Fig. 1). Indeed, increment of value $\Gamma$ leads to grows of the function $\varepsilon^{\prime}\left(\omega_{\min }\right)$ value with simultaneously narrowing of the negative DP bandwidth. It is shown on Fig.2. If $\varepsilon^{\prime}\left(\omega_{\min }\right)=0$ for certain "critical" damping (curve 2 on Fig.2) then negative DP band is fully disappeared. So for the existence of negative permittivity region where $\varepsilon^{\prime}(\omega)<0$ it is necessary to:

$$
\varepsilon^{\prime}\left(\omega_{\min }\right)=\varepsilon^{\infty}-\frac{S \omega_{T}^{2}}{2 \omega_{T} \Gamma-\Gamma^{2}}<0 .
$$

It is resulted to the following inequality:

$$
\Gamma^{2}+2 \omega_{T} \Gamma-\frac{S}{\varepsilon^{\infty}} \omega_{T}^{2}<0
$$
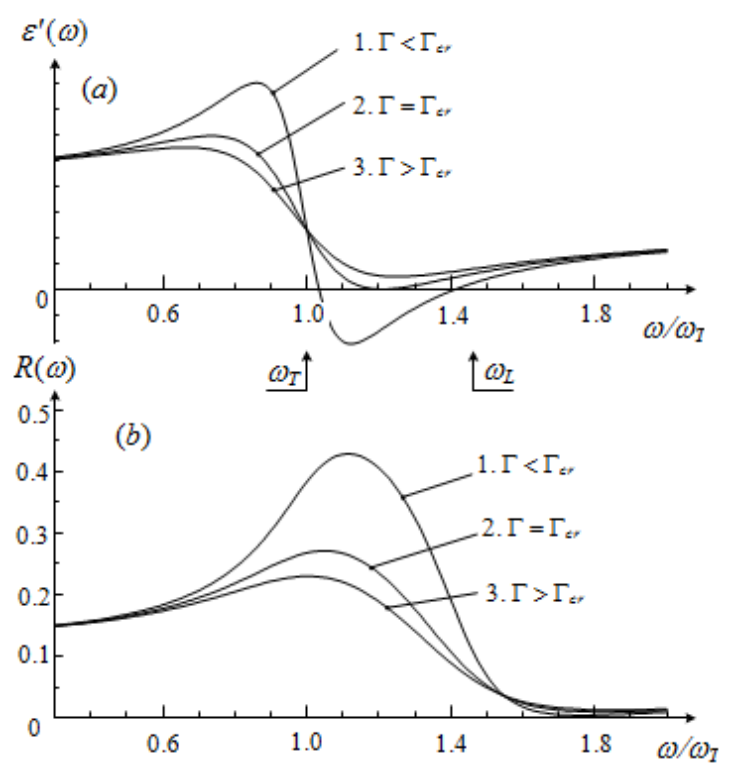

Fig 2. Dielectric function $\mathcal{E}^{\prime}(\omega)$ (a) and infrared reflection spectra (b) with phonons attenuation close to the critical value defined by (17). The negative DP region disappears at $\Gamma=\Gamma_{c r}$ and it can exist only for $\Gamma<\Gamma_{c r}$

Excluding the $\Gamma$ term from inequality (13) for interval $\Gamma>$ 0 we obtain the condition that restricts the damping constant:

$$
\Gamma<\omega_{T}\left(\sqrt{\frac{\varepsilon_{0}}{\varepsilon^{\infty}}}-1\right) .
$$

Consequently within the model of single oscillator crystal we have obtained the fairly simple quantitative criterion for the existence of frequency bands with negative DP. This criterion is based on the ratio of the phonon damping constant to the frequency $\omega_{T}$ and it contains only material parameters of crystal such as $\varepsilon_{0}$ and $\varepsilon^{\infty}$. 
As $S=\varepsilon_{0}-\varepsilon^{\infty}$ the inequality (14) can be represented in an equivalent form for the oscillator strength $S$ :

$$
S>\varepsilon^{\infty} \frac{\Gamma}{\omega_{T}}\left(\frac{\Gamma}{\omega_{T}}+2\right) .
$$

This second form of our criteria for the oscillator strength $S$ is expressed in terms of relative phonon attenuation $\Gamma / \omega_{T}$. Inequality (15) gives a lower limit for the oscillator strength of the polar vibrations when negative DP is created by this phonon.

Finally, the substitution LST ratio (1) in the expression (14) leads to a further alternative form of our criterion:

$$
\Gamma<\left(\omega_{L}-\omega_{T}\right) \text { or } \frac{\Gamma}{\omega_{L}-\omega_{T}}<1 .
$$

The inequality (16) is directly resulted the region of negative permittivity. It exists until the phonon attenuation constant does not exceed T-L splitting for this phonon.

Thus, it is useful to define the critical damping $\Gamma_{c r}$ value using the inequalities (14) and (16):

$$
\frac{\Gamma_{c r}}{\omega_{T}}=\sqrt{\frac{\varepsilon_{0}}{\varepsilon^{\infty}}}-1=\frac{\omega_{L}}{\omega_{T}}-1
$$

as an upper limit of the phonon damping and it enclose the area of negative DP existence in the crystal.

Minimum of dielectric function $\mathcal{E}^{\prime}(\omega)$ is equal to zero for critical damping as one can see on Fig. 2a (curve 2). All material parameters except $\Gamma$ in our calculation for Fig. 2 are corresponded to the crystal $\mathrm{KBr}$ with one polar vibration.

Certain frequency band with negative DP values around the minimum frequency $\omega_{\min }$ is formed in the case of $\Gamma<\Gamma_{c r}$ and it is located within the T-L frequency splitting of this oscillation. In contrast function $\mathcal{E}^{\prime}(\omega)$ is positive everywhere in the case of $\Gamma>\Gamma_{c r}$ (curve 3 on Fig. 2a) including the residual ray band $(\mathrm{T}-\mathrm{L})$.

Note the reflectance spectrum on Fig. $2 b$ doesn't show any characteristic changes just pointing to the presence or absence of the negative DP region. Thus it is necessary to apply full DA processing of the IR measurements data to restore the dielectric function $\mathcal{E}^{\prime}(\omega)$ and our criterion may be used to significantly simplify this analysis of negative DP.

Moreover if polar vibration is active in Raman spectrum than the presence or absence of negative DP band may be determined directly using our criterion. It's enough to first-hand define the fundamental frequencies of transverse $\omega_{T}$ and longitudinal $\omega_{L}$ vibrations from the appropriate Raman lines position in the spectrum and damping constant $\Gamma$ is found as half-width of corresponding Raman line. At last the presence of negative DP band is determined by a simple test of inequality (16).

\subsection{Negative DP Bandwidth}

The frequency range of negative DP area really doesn't match to the T-L splitting, unlike the idealized case of no damping, which describes the Kurosawa ratio (2). Negative DP area is shrinking relative to T-L splitting up to its complete disappearance by damping constant increasing, as shown in Fig. 1 (b). Therefore, we have studied behavior of the frequency band of negative DP as function of the damping constant parameter.

Let introduce the notation of the frequencies: $\omega^{-}$is the frequency when DP starts to take negative values, and $\omega^{+}$is the frequency when DP out from negative region. If one consider the case of single oscillator model $(N=1)$ for expression (3) and substitution of the resulting $S$ value in the first equation of system (6), then it is obtain:

$$
\varepsilon^{\prime}(\omega)=\varepsilon^{\infty}\left[1+\frac{\left(\omega^{2}-\omega_{T}^{2}\right)\left(\omega_{T}^{2}-\omega_{L}^{2}\right)}{\Gamma^{2} \omega^{2}+\left(\omega^{2}-\omega_{T}^{2}\right)^{2}}\right] .
$$

The frequencies $\omega$ and $\omega^{+}$are found in terms of equality of the real DP part described by expression (18) to zero $\varepsilon^{\prime}(\omega)$ $=0$. After simple mathematical transformations and rejecting no physical variations we obtain:

$$
\begin{gathered}
\omega^{ \pm}=\frac{1}{\sqrt{2}}\left(\omega_{L}^{2}+\omega_{T}^{2}-\Gamma^{2} \pm\right. \\
\left.\quad \pm \sqrt{\left(\omega_{L}^{2}+\omega_{T}^{2}-\Gamma^{2}\right)^{2}-4 \omega_{L}^{2} \omega_{T}^{2}}\right)^{1 / 2} .
\end{gathered}
$$

Let $\Delta \omega=\omega^{+}-\omega^{-}$is the real frequency band of negative DP. Explicit expression for $\Delta \omega$ may be easily founded using the relations (19), so we have:

$$
\Delta \omega=\left(\omega_{L}-\omega_{T}\right) \sqrt{1-\left(\frac{\Gamma}{\omega_{L}-\omega_{T}}\right)^{2}} .
$$

The expression (20) is the generalization of no damping idealized case as if $\Gamma=0$ then $\Delta \omega=\omega_{L}-\omega_{T}$. Similarly, if the damping constant is directed to zero in expressions (19) then we get: $\omega^{-}=\omega_{T}$, and $\omega^{+}=\omega_{L}$.

Our criterion and single oscillation model may be applied to crystals with one pronounced vibration in the infrared region without any restrictions. Such objects are alkali-halide crystals ( $\mathrm{NaCl}, \mathrm{KBr}, \mathrm{NaF}, \mathrm{LiF}$ and many others) and very significant negative DP in absolute value is observed in this crystals. Typical difference $\omega_{L}-\omega_{T}$ can be reached up to several hundreds of $\mathrm{cm}^{-1}$ for such crystals whereas damping $\Gamma$ is not exceeding of a few tens of $\mathrm{cm}^{-1}$ at room and low temperatures. Thus $\Gamma /\left(\omega_{L}-\omega_{T}\right)<0,1$ and it is ensures in accordance with (16) the existence of large negative DP values as it is observed in practice. The frequency range of $\mathcal{E}^{\prime}(\omega)<0$ almost coincides with the interval $\omega_{T}<\omega<\omega_{\mathrm{L}}$ in this case.

It should be noted the application of our criterion is substantially limited by restrictions of the single oscillation model only in the first form (14) since it is contained the material parameters $\varepsilon_{0}$ and $\varepsilon^{\infty}$. Alternatively the third 
criterion form (16) is independent from material parameters and exclusively vibration parameters $\omega_{L}, \omega_{T}$ and $\Gamma$ terms are implied. So we assume the alternative criterion forms may be used to analysis of negative dielectric properties in the great number of more complex crystalline solids with multi-phonon spectrum.

Below we present the test results of negative dielectric properties in the model crystal of boron nitride BN.

\section{Test Results in BN and Discussion}

The boron nitride BN crystal is of interest for test our results due the presence of three crystal modifications specifically cubic, orthorhombic and hexagonal. Boron nitride BN crystals were grown relatively recently, and their phonon spectra sufficiently investigated in [15].

Table 1. Material parameters and polar vibrations characteristics in boron nitride of cubic, orthorhombic and hexagonal crystals modifications

\begin{tabular}{|c|c|c|c|c|c|c|c|}
\hline vibration number & Structure & Orientation & $\varepsilon^{\infty}$ & $\mathbf{S}$ & $\omega_{T}\left(\mathrm{~cm}^{-1}\right)$ & $\omega_{L}\left(\mathrm{~cm}^{-1}\right)$ & $\Gamma\left(\mathrm{cm}^{-1}\right)$ \\
\hline & 2 & 3 & 4 & 5 & 6 & 7 & 8 \\
\hline 1 & Cubic & & 4,6 & 2,40 & 1057 & 1306 & 5,39 \\
\hline 2 & Orthorhombic & $\vec{k} \| \vec{C}$ & 5,2 & 2,00 & 1374 & 1617 & 6,87 \\
\hline 3 & & & & 0,34 & 795 & 829 & 7,47 \\
\hline 4 & & $E \perp C$ & 3,2 & 0,51 & 1498 & 1616 & 80,89 \\
\hline 5 & Hexagonal & & & 0,22 & 779 & 794 & 49,08 \\
\hline 6 & & $\vec{k} \| \vec{C}$ & 3,8 & 1,10 & 1373 & 1487 & 31,58 \\
\hline 7 & & & & 0,13 & 1508 & 1615 & 102,54 \\
\hline
\end{tabular}

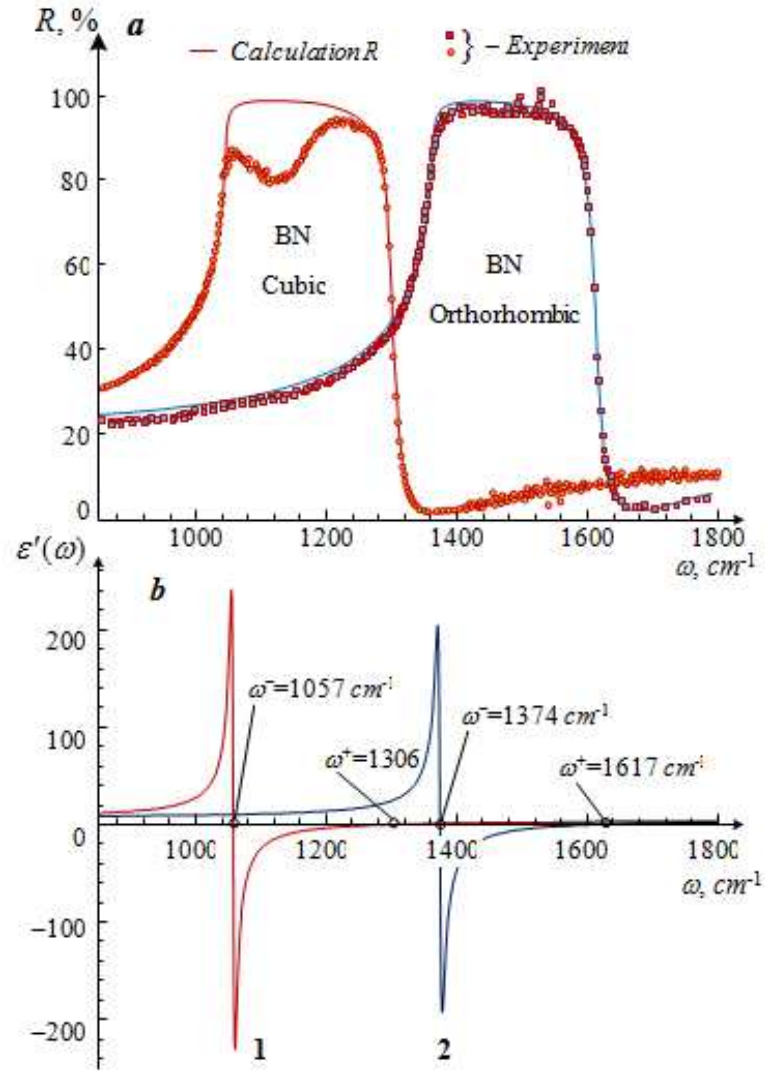

Fig 3. (a) IR reflection spectra (after [15]). (b) Our calculation of frequency dispersion $\varepsilon^{\prime}(\omega)$ for the cubic (1) and orthorhombic (2) BN systems. The negative DP narrowing can be neglected in comparison with the frequency T-L splitting for both crystal modifications.

We have analyzed the variance $\varepsilon^{\prime}(\omega)$ near seven fundamental vibrations for different crystal systems of BN structure (1 cubic, 1 orthorhombic and 5 hexagonal). These modes were observed at different orientations of the wave vector $\vec{k}$ and the electric field vector $\vec{E}$ of the electromagnetic wave relatively to the crystal $\mathrm{C}$ axis.
$\mathrm{C}$ axis direction is the preferred orientation of the crystallographic axes and it is coincides with the normal to the sediment-crystal growth. The dumping constant $\Gamma, T$ and $\mathrm{L}$ frequencies are given in Table 1 after the data of [15]. The oscillator strengths $S$ (column 5) was calculated by us using (4).

There is different number of polar oscillations in the IR reflection spectrum depending on the BN crystal symmetry. Only one oscillation is appeared both in cubic and in orthorhombic crystal modifications. These cases may be directly analyzed in accordance with single mode approach and our results are illustrated using Fig.3.

In contrast several fundamental modes can be observed in the hexagonal BN crystal symmetry for appropriate orientation of wave vector $\vec{k}$ and/or polarization of vector $\vec{E}$ with respect to $\mathrm{C}$ axis. Namely two polar vibrations are appeared in experimental geometry $\vec{E} \perp C$ (see Fig.4a) and three polar vibrations are seen in the orientation $\vec{k} \| C$ (Fig.5a). Thus the spectral analysis of the hexagonal BN system allows us to determine the applicability limits of the criterion for the complex spectra case with multiple polar modes.

Table 2. The relative damping $\gamma=\Gamma /\left(\omega_{L}-\omega_{T}\right), \quad \varepsilon_{\min }^{\prime}$, and negative $D P$ bandwidth in BN crystal

\begin{tabular}{lllllll}
\hline No.e & $\begin{array}{l}\left(\boldsymbol{\omega}_{\boldsymbol{L}}-\boldsymbol{\omega}_{\boldsymbol{I}}\right) \\
\mathbf{c m}^{-1}\end{array}$ & $\boldsymbol{\gamma}$ & $\omega_{0}^{-}, \mathbf{c m}^{-1}$ & $\omega_{0}^{+}, \mathbf{c m}^{-1}$ & $\boldsymbol{\varepsilon}_{\min }^{\prime}$ & $\begin{array}{l}\omega_{0}^{+}-\omega_{0}^{-} \\
\mathbf{c m}^{-1}\end{array}$ \\
\hline 1 & 249 & 0,02 & 1057,02 & 1305,73 & -232 & 248,71 \\
2 & 243 & 0,03 & 1374,04 & 1616,73 & -194 & 242,69 \\
3 & 34 & 0,22 & 795,41 & 828,59 & -14 & 33,18 \\
4 & 118 & 0,69 & 1513,38 & 1599,57 & $-1,4$ & 86,19 \\
5 & 15 & 3,27 & No negative DP region & & \\
6 & 114 & 0,28 & 1374,46 & 1604,57 & -19 & 230,11 \\
7 & 107 & 0,96 & & & $-1,5$ & 23,11 \\
\hline
\end{tabular}

The DP real parts $\varepsilon^{\prime}$ we have calculated for all phonon oscillations presented in Table 1. Results of our quantitative analysis of damping effect on the negative DP are collected in the Table 2. The relative damping $\gamma$ is determined as 
$\gamma=\Gamma /\left(\omega_{L}-\omega_{T}\right)$ and its value is directly used for the criterion of the negative DP existence in form of inequality (16). Both characteristic frequencies $\omega_{0}^{-}$and $\omega_{0}^{+}$was calculated using (19) and its values (if exist) are presented accordantly in $4^{\text {th }}$ and $5^{\text {th }}$ columns of the Table 2 . Sufficiently small attenuation $(\Gamma \approx 0)$ leads to approximate equality $\omega_{0}^{-} \approx \omega_{T}$ and $\omega_{0}^{+} \approx \omega_{L}$. It is fully matched to theoretical prediction based on (19) and (20). The real narrowing degree of negative DP band $\Delta \omega=\omega_{0}^{+}-\omega_{0}^{-}$is followed by relative damping value $\gamma$ until $\gamma<1$.

The frequency dispersion relations $\varepsilon^{\prime}(\omega)$ are shown on Fig. 3b, 4b, 5b for all studied $\mathrm{BN}$ crystal structures. Continuous numbering of vibration we use in both tables and figures 3-5. It is marked by the corresponding numeric symbols below each dispersion curves on figures.

Single fundamental oscillation is appeared with large frequency shift of $\omega_{T}$, exceeding $300 \mathrm{~cm}^{-1}$ at crystal symmetry is lowered from the cubic system to the orthorhombic modification (Fig. 3). But T-L frequency splitting is almost equals $\sim 250 \mathrm{~cm}^{-1}$ for both cubic modification and orthorhombic system (orientation $\vec{k} \| C$ ) in the boron nitride crystal.

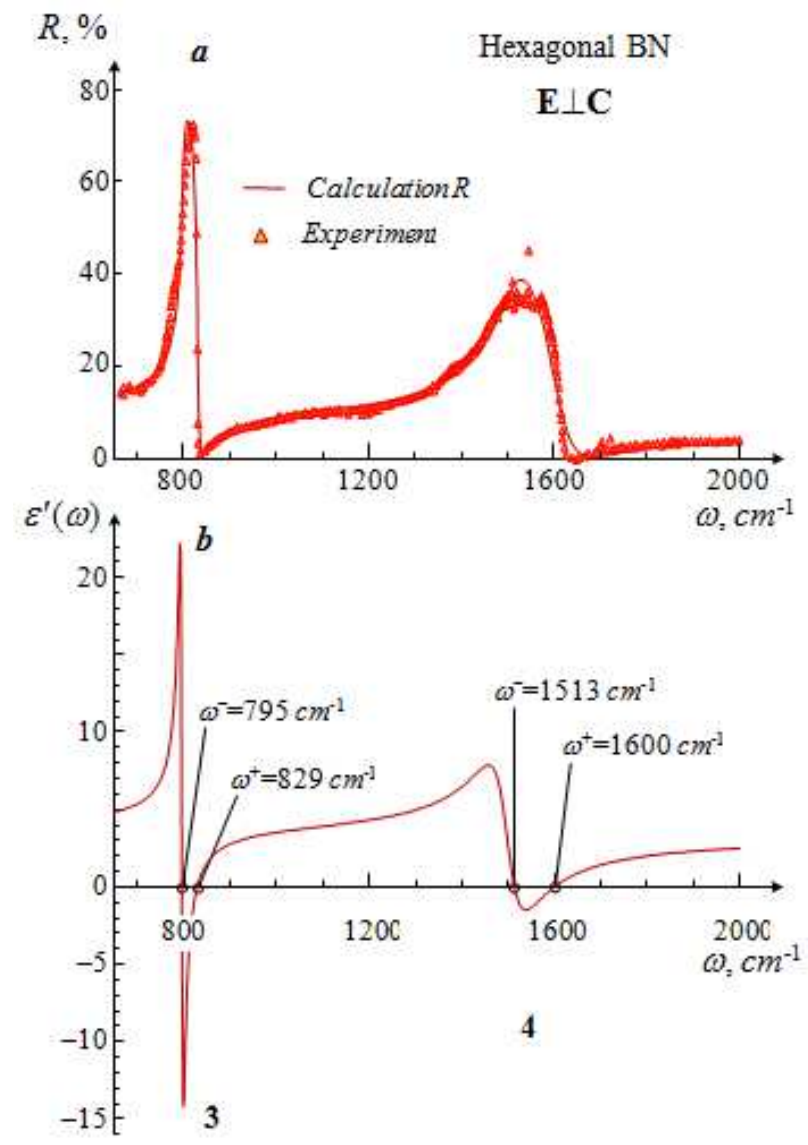

Fig 4. (a) Experimental (triangles) and calculated (solid line) IR reflection spectrum of hexagonal $B N$ in the experimental orientation $\vec{E} \perp C[15]$ (b) Frequency dispersion $\varepsilon^{\prime}(\omega)$. Dumping effect on the negative DP narrowing is especially noticeable for mode no. 4 as its relative attenuation is close to unity $(\gamma \approx 0.7)$.

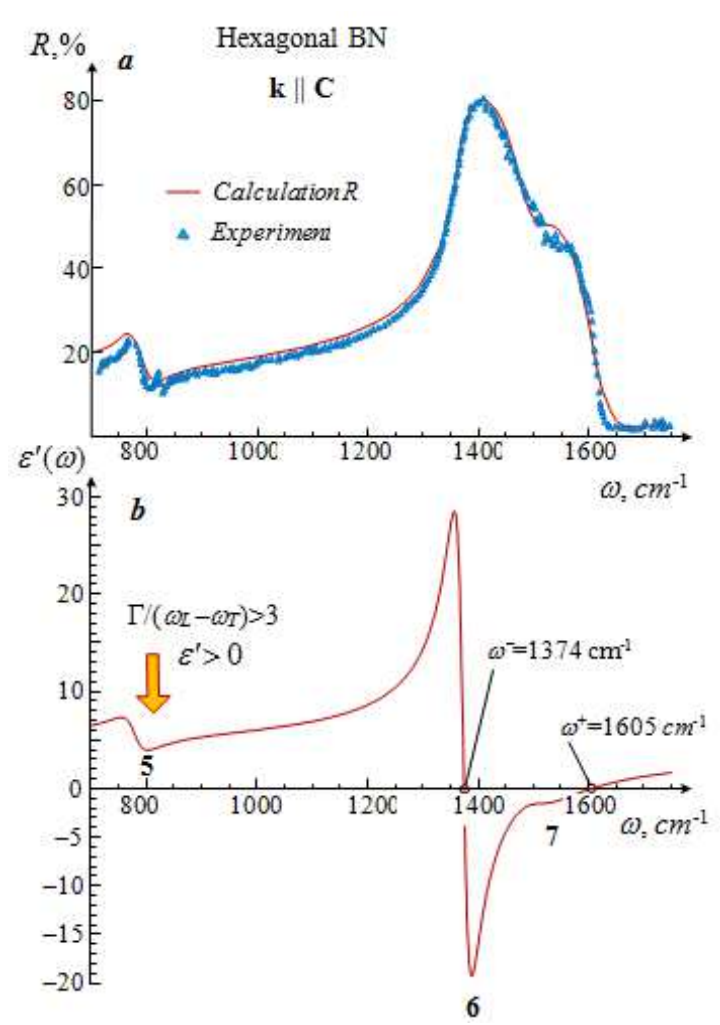

Fig 5. (a) Experimental (triangles) and calculated (solid line) IR reflection spectrum of hexagonal $B N$ in geometry $\vec{k} \| C$ [15]; (b) Frequency dispersion $\varepsilon^{\prime}(\omega)$. Too dumped mode no.5 does not create the negative DP area as $\gamma>1$. Unity of modes no. 6 and no. 7 get two picks $\varepsilon_{\min }^{\prime}<0$ since $\gamma$ $<1$ for both vibrations.

The same attenuation of the fundamental vibrations in absolute terms is about of $<10 \mathrm{~cm}^{-1}$. Thus relatively T-L splitting $\gamma$ is equal to 0.02 for phonon of cubic system and it is $\gamma=0.03$ for orthorhombic modification as indicated in the Table 2. As result the negative DP narrowing can be neglected in full accordance with the criteria (14) and (16) because it is only on $\sim 0.3 \mathrm{~cm}^{-1}$ less than the T-L frequency splitting. Simultaneously $\varepsilon_{\min }^{\prime} \approx-200$ in both cases and it is almost by 40 times the value of low-frequency dielectric constant of crystalline compounds.

The presence of several phonon vibrations in hexagonal BN spectra actually does not allow to direct use of the criterion (14). However the presence of experimentally determined data $\omega_{T}$ and $\omega_{L}$ was for us the basis for formal application of criteria form (16). Indeed the observable negative DP narrow $\sim 1 \mathrm{~cm}^{-1}$ for mode no. 3 with $\omega_{T}=795$ $\mathrm{cm}^{-1}$ in the orientation of $\vec{E} \perp C$ (see Fig. 4) is associated with significantly higher (by 10 times) than in the previous as the relative attenuation $\gamma=0.22$.

Simultaneously, the minimum value $\varepsilon_{\min }^{\prime} \approx-14$ for this mode also by the order of magnitude is decreased in absolute value in comparison to the previous cases of cubic and orthorhombic modifications.

Even more brightly phonon damping effect is associated with vibration of $\omega_{T}=1498 \mathrm{~cm}^{-1}$ (Fig. 4, mode no.4). The substantially higher relative attenuation factor $\gamma=0.69$ does 
not only reduced the absolute value $\varepsilon_{\min }^{\prime}$ to -1.4 , but it also is lowered the negative DP region on more than $30 \mathrm{~cm}^{-1}$ as would be predicted using our criterion in form (16).

Our analysis of three phonons in spectrum of hexagonal $\mathrm{BN}$ modification at orientation $\vec{k} \| C$ gives the dispersion curve $\mathcal{E}^{\prime}(\omega)$ and it is shown on Fig.5b. The relative attenuation is $\gamma=3.3$ for too damped mode no. 5 , i.e. it is three times the critical value. The variance $\mathcal{E}^{\prime}$ is leaved quite positive for this mode without the formation of any negative DP area and it again is in full compliance with the criterion (16).

The existence of negative DP values $\varepsilon_{\min }^{\prime}$ for vibrations no. 6 and no. 7 is confirmed by our criterion (16) since the relative attenuation are 0.28 and 0.96 respectively. In addition they are really appeared on Fig. $5 \mathrm{~b}$ with individual values: $\varepsilon_{\min }^{\prime}=-19$ and $\varepsilon_{\min }^{\prime}=-1.5$. However, there are some features at the formation of negative DP band in the last case. Significant overlap are associated with one phonon line at frequency $\omega_{T}=1373 \mathrm{~cm}^{-1}$ and second line at frequency $\omega_{T}=$ $1508 \mathrm{~cm}^{-1}$. The result there is the union of two overlapping bands in the common frequency interval of negative DP with $\Delta \omega=230 \mathrm{~cm}^{-1}$ from $\omega_{0}^{-}=1374.5 \mathrm{~cm}^{-1}$ to $\omega_{0}^{+}=1604.6 \mathrm{~cm}^{-1}$. The start frequency $\omega_{0}^{-}$of this common negative DP interval is shifted on $1.5 \mathrm{~cm}^{-1}$ from $\omega_{T}$ for the first phonon and end frequency $\omega_{0}^{+}$is located on about of $10 \mathrm{~cm}^{-1}$ lower than the corresponding second phonon frequency $\omega_{L}$.

Thus, the introduced criterion allows us to clearly predict the existence of negative DP region based on spectroscopic parameters of single mode spectrum and its form (16) may be useful for the analysis of particular complex IR reflection spectra.

\section{Conclusion}

In summary, it is obtained the boundary limits of negative DP existence in real crystalline media due to oscillation damping effects in this work. Damping effect on the negative DP area is shrinking relative to T-L splitting up to its complete disappearance. The critical damping $\Gamma_{c r}$ value is defined in our paper as an upper limit of the phonon damping and it enclose the area of negative DP existence in the crystal. The physical meaning of $\Gamma_{c r}$ is zeroing of the dielectric function $\mathcal{E}^{\prime}(\omega)$ minimum.

The simple quantitative criterion for the negative DP existence is given based on single oscillation model. Our criterion is presented in three equivalent forms as inequalities between the fundamental crystal constants and spectroscopic parameters of polar vibration. All three forms of our criterion are characterized by simplicity of its analytical expressions and the practical application.

Criterion applicability to multimode case is verified by set of vibrations testing in three crystalline modifications of boron nitride. We expect the proposed criteria of negative DP existence may be specifically useful in the studies of noncentrosymmetric crystals when the value $\omega_{T}, \omega_{L}$ and $\Gamma$ available for direct measurement from Raman spectra.

\section{Acknowledgement}

The authors thank our teacher prof. P.A. Korotkov for his support and regular stimulation to this work.

\section{References}

[1] V. G. Veselago, "The electrodynamics of substances with simultaneously negative values of $\varepsilon$ and $\mu$," Sov. Phys. Usp. vol.10, no,3, pp,509-514, 1968.

[2] V.M. Agranovich, Yu.N. Gartstein. "Spatial dispersion and negative refraction of light," Physics - Uspekhi, vol. 49 no.10, pp.1051-1068, 2006.

[3] V.G. Veselago, "Energy, momentum and mass transfer by an electromagnetic wave in a negative refraction medium," Physics - Uspekhi, vol. 52, no.6, pp. 689-694, 2009.

[4] S. Ornes, "Metamaterials," Proceedings of the National Academy of Sciences, vol. 110, no. 21 p. 8319, 2013 (http://www.pnas.org/content/110/21/8319)

[5] J. B. Pendry, D. Schurig, and D. R. Smith, "Controlling electromagnetic fields," Science, vol. 312, pp.1780-1782, June 2006.

[6] U. Leonhardt, "Optical conformal mapping," Science, vol. 312, pp.1777-1780, June 2006.

[7] A.V. Kildishev, V.M. Shalaev, Enabling transformation optics through metamaterials. Physics - Uspekhi, vol. 54, no.1, 2011.

[8] D. Schurig, J. J. Mock, B. J. Justice, S. A. Cummer, J. B. Pendry, A. F.Starr, and D. R. Smith, "Metamaterial electromagnetic cloak at microwave frequencies," Science, vol. 314, pp.977-980, November 2006.

[9] R. Liu, C. Ji, J. J. Mock, J. Y. Chin, T. J. Cui, and D. R. Smith, "Broadband ground-plane cloak," Science, vol. 323, pp.366-369, January 2009.

[10] J.B. Pendry, "Negative refraction makes a perfect lens," Phys. Rev. Lett., vol. 85, pp. 3966-3969, 30 October 2000.

[11] R. H. Lyddane, R. G. Sachs, E. Teller, "On the polar vibrations of alkali halides," Phys. Rev., vol.59, no.8, pp. 673-676, 1941 .

[12] Tatsumi Kurosawa, "Polarization waves in solids," J. Phys. Soc. Jap., vol.16, no.7, pp. 1288-1308, 1961.

[13] Max Born and Kun Huang, "Experimental aspect of infra-red dispersion by ionic crystals," pp.116-128, in Dynamical theory of crystal lattices. Oxford University Press, Amen House, London E.C.4, 1954.

[14] A.S. Barker, R. Loudon, "Response functions in the theory of Raman scattering by vibrational and polariton modes in dielectric crystals," Rev. Mod. Phys., vol.41, pp.18-47, January 1972.

[15] S.V. Ordin, B.N. Sharupin , M.I. Fedorov, "Normal lattice oscillations and crystal structure of anisotopic modifications of boron nitride," Semiconductors [Physics and Technics Semiconductors], vol. 32, no.9, pp. 1033-1042, 1998. 